> Le cytomégalovirus humain (CMVH) est un pathogène opportuniste majeur en cas d'immunodépression et représente la principale cause d'infection congénitale d'origine virale. Bien qu'efficace, l'utilisation des molécules conventionnelles est limitée par leur toxicité et par l'émergence de résistance du virus, rendant nécessaire le développement de nouveaux traitements. Lors de la réplication du CMVH, l'encapsidation de l'ADN est réalisée par le complexe terminase qui clive l'ADN pour empaqueter le génome dans la capside. L'absence d'homologues dans les cellules des mammifères rend les protéines du complexe terminase des cibles idéales pour des antiviraux spécifiques. Une nouvelle molécule, le letermovir, cible une étape exclusivement virale en interagissant avec le complexe terminase. Son efficacité a été prouvée lors d'essais cliniques de phase III. Néanmoins, son mécanisme d'action n'est, à ce jour, pas élucidé et aucune activité n'est observée contre les autres herpèsvirus. <

\section{Les traitements actuels de l'infection à cytomégalovirus et l'émergence de résistance}

Le cytomégalovirus (CMVH) est un bêta-herpèsvirus ubiquitaire infectant 50 à $100 \%$ de la population mondiale, la prévalence variant en fonction du niveau socio-économique. La primo-infection, souvent asymptomatique chez les personnes immunocompétentes, est suivie d'une phase de latence au cours de laquelle peuvent survenir des réactivations. Le CMVH représente l'agent pathogène le plus fréquemment rencontré en transplantation : 50 à $70 \%$ des patients présentent une primo-infection ou une réactivation dans l'année qui suit la greffe $[1,2]$. Il est également la première cause d'infection congénitale virale dans le monde. Alors que dans les pays industrialisés la prévalence est comprise entre 0,3 et $1,2 \%$, elle est plus difficile à évaluer dans les

\title{
Le complexe terminase, une cible de choix dans le traitement de l'infection à cytomégalovirus humain
}

Gaëtan Ligat ${ }^{1,2, *}$, Clotilde Muller ${ }^{1,2}$, Sophie Alain ${ }^{1,2}$, Sébastien Hantz ${ }^{1,2}$

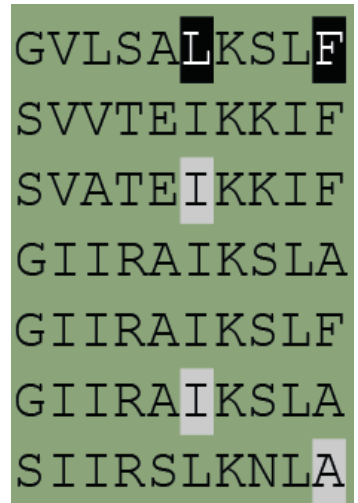

pays en développement. Néanmoins, on estime que $1 \%$ des enfants dans le monde sont infectés par le CMVH in utero.

Les antiviraux actuellement disponibles en pratique clinique sont peu nombreux. Le ganciclovir (GCV), et sa prodrogue orale, le valganciclovir (VGCV), le cidofovir (CDV) et le foscarnet (FOS) inhibent l'activité de I'ADN polymérase virale pUL54 (Figure 1). Bien qu'efficaces, ces molécules présentent une toxicité hématologique et/ou rénale, et sont à l'origine de l'émergence de mutations de résistance [3]. De par leur toxicité, leur utilisation est contre-indiquée chez la femme enceinte. L'aciclovir (ACV) ou sa prodrogue orale, le valaciclovir (VACV), ciblent également I'ADN polymérase virale. Ils peuvent être utilisés chez les transplantés, mais uniquement en prophylaxie en raison de leur faible efficacité contre le virus, ou en cas d'infection congénitale, du fait de leur absence de toxicité [4].

De nouvelles molécules présentant une meilleure biodisponibilité orale et une moindre toxicité sont en cours de développement. Le letermovir (ou PREVYMISTM) a reçu en novembre 2017 l'approbation de l'Europe et des États-Unis pour une utilisation en prophylaxie dans le cadre de greffes de cellules souches hématopoïétiques. Cette molécule cible une étape exclusivement virale, l'encapsidation du génome (Figure 1). 


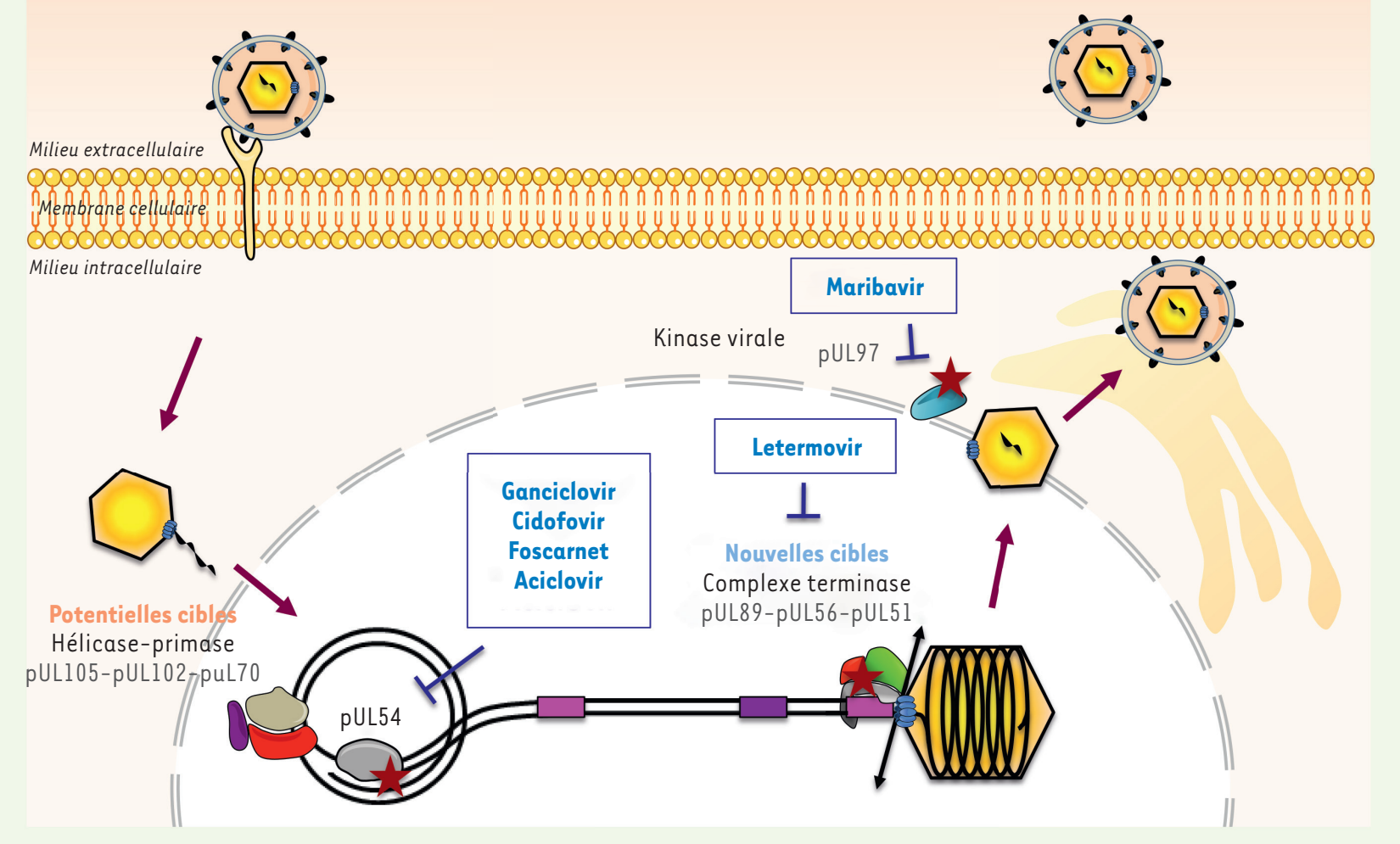

Figure 1. Cycle de réplication du CMVH et site d'action des molécules antivirales. Après l'attachement du virus à la cellule, la fusion des membranes cellulaire et virale aboutit à la pénétration du virus qui est transporté via le réseau des microtubules jusqu'au noyau où l'ADN viral est libéré et circularisé. La réplication, selon le modèle du cercle roulant, dépend de plusieurs protéines et plus particulièrement du complexe hélicase-primase (pUL105-pUL102- pUL70) et de l'ADN polymérase pUL54. pUL56, pUL89 et pUL51, formant un hétéro-oligomère nommé complexe terminase, participant à l'encapsidation du génome viral. La sortie du virion ainsi formé nécessite la kinase virale pUL97. Le virion est alors transporté dans le cytoplasme via le réseau des microtubules, permettant sa maturation et son enveloppement dans l'appareil de Golgi avant son excrétion. Les antiviraux actuels (ganciclovir, cidofovir, foscarnet et aciclovir) interfèrent avec l'activité de la polymérase virale pUL54. Le maribavir a pour cible la kinase virale pUL97 et le letermovir agit sur l'étape d'encapsidation du génome viral en interagissant avec le complexe terminase. Les mutations associées à une résistance aux actuels antiviraux sont signalées par une étoile.

\section{Le complexe terminase : des protéines indispensables à l'étape d'encapsidation}

La réplication du génome du CMVH aboutit à la formation de concatémères d'ADN qui agissent comme substrat lors du processus d'encapsidation (Figure 1). Un complexe de protéines virales, appelé «complexe terminase», permet la reconnaissance, le clivage et la translocation des unités de génome dans des capsides néoformées [5]. Le génome du CMVH comporte, à ses extrémités et en son centre, des séquences répétées, appelées séquences « $a$ », qui contiennent des éléments cis-régulateurs ( pac» ou «cis-acting packaging element»). Le processus d'encapsidation implique un clivage sitespécifique au niveau de séquences riches en AT des éléments «pac ». Le complexe terminase, hétéro-oligomérique, est constitué des sousunités pUL56, pUL89 et pUL51 [6,7]. D'autres protéines, pUL52, pUL77 et pUL93, ont également été identifiées comme des partenaires du complexe [8-12]. Après sa translocation dans le noyau, le complexe terminase reconnaît les séquences «pac», effectue un premier clivage de I'ADN viral, et recrute une capside vide. II réalise alors un second clivage libérant l'ADN viral dans la capside via une interaction avec les protéines pUL104. Le complexe se dissocie finalement, permettant une nouvelle encapsidation [13].

\section{La grande sous-unité pUL56}

pUL56, aussi nommée grande sous-unité du complexe terminase, est une protéine de 850 acides aminés codée par un gène de 2553 paires de bases $(\mathrm{pb})$ situé dans le segment unique long du génome du virus. D'environ $130 \mathrm{kDa}$, 


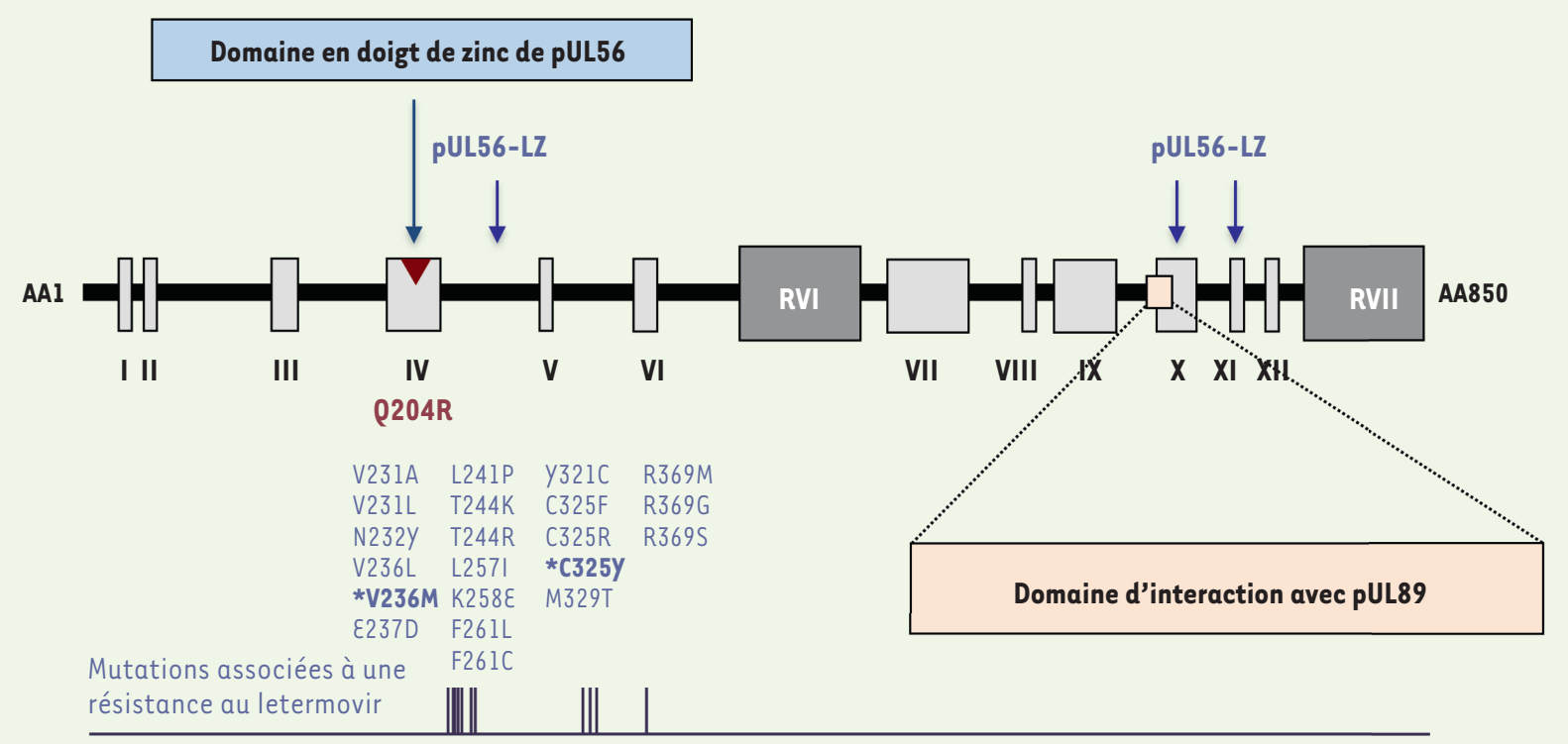

Régions conservées :

\begin{tabular}{|c|c|c|c|}
\hline I - M21-P31 & IV - C191-I220 & VII - E514-R572 & $X-y 676-F 713$ \\
\hline II - F41-062 & $V-0272-W 300$ & VIII - Y590-L600 & XI - V732-F744 \\
\hline III - L134-E141 & VI - $\varepsilon 356-1374$ & IX - I617-L658 & XII - D755-L764 \\
\hline
\end{tabular}

Régions variables :

VRI - A425-E485

VRII - V778-R850

Figure 2. Régions conservées de la sous-unité pUL56. pUL56 est composé de 12 régions conservées (I-XII). La région conservée IV représente un domaine doigt de zinc (DZ). La partie centrale de pUL56 et l'extrémité C-terminale comprennent deux régions variables annotées RVI et RVII. Les trois «Leucine zipper», annotées pUL56-LZ, sont indiquées. La séquence située en C-terminale de pUL56 ( ${ }_{671}$ WMVVKYMGFF $_{680}$ ), essentielle pour I'interaction avec pUL89, est indiquée. Les positions des acides aminés associées à la résistance in vitro au letermovir sont spécifiées [35, 36]. Les mutations associées à des résistances identifiées lors d'études cliniques sont signalées en gras par une étoile (www.accessdata.fda.gov, Reference ID 4179078). La position de la mutation associée à la résistance au benzimidazole (Q204R) est indiquée en rouge (adaptée de [33]).

elle est constituée de 12 régions conservées (Figure 2) [14]. pUL56 est hautement conservée. Elle a été identifiée et caractérisée en partie grâce à son homologie avec la protéine pUL28 d'HSV-1 (herpes simplex virus 1) [15,16]. Elle présente la capacité de s'auto-dimériser [17].

pUL56 est impliquée, notamment, dans l'activité ATPase du complexe terminase et la reconnaissance de motifs cis-régulateurs «pac», nécessaire au clivage des concatémères d'ADN, produits de la réplication virale [18]. Bogner et ses collaborateurs ont en effet mis en évidence l'interaction spécifique de pUL56 avec des motifs «pac» [19]. Le processus d'encapsidation de l'ADN viral est initié dans des structures nucléaires appelées «centres de réplication», dans lesquelles pUL56 s'accumule tardivement après l'infection, et s'associe aux protéines pUL112, pUL113 et pUL44, trois protéines impliquées dans la réplication virale. L'importation de pUL56 dans le noyau est réalisée par une voie importine, par l'interaction de la protéine hSRPla ${ }^{1}$ avec le signal de localisation nucléaire (NLS) situé dans la

${ }^{1}$ Protéine reconnaissant le signal de translocation nucléaire et permettant à la protéine exprimant ce signal de pénétrer dans le noyau. partie C-terminale de pUL56 (résidus d'acides aminés 816 à 827). Une analyse mutationnelle a permis de préciser que l'arginine 822 et la lysine 823 étaient les résidus essentiels du signal de localisation de pUL56 [20]. Contrairement à pUL56 qui peut se localiser seule dans le noyau, les sous-unités pUL89 et pUL51 nécessitent la présence simultanée des trois sousunités du complexe (pUL56, pUL89, pUL51) pour leur translocation nucléaire [21]. Une interaction spécifique entre la partie C-terminale de pUL56 et pUL89 a d'ailleurs été mise en évidence par co-immunoprécipitation [22] et nous avons montré qu'une courte séquence de dix résidus localisés dans la région C-terminale de pUL56 $\left({ }_{671}\right.$ WMVVKYMGFF $\left._{680}\right)$ était essentielle pour cette interaction [23]. pUL89 est responsable de la première étape du clivage de l'ADN concatémère qui est suivie de la liaison du complexe terminase aux séquences «pac». Lors du processus d'encapsidation de l'ADN, pUL56 interagit également avec la protéine 

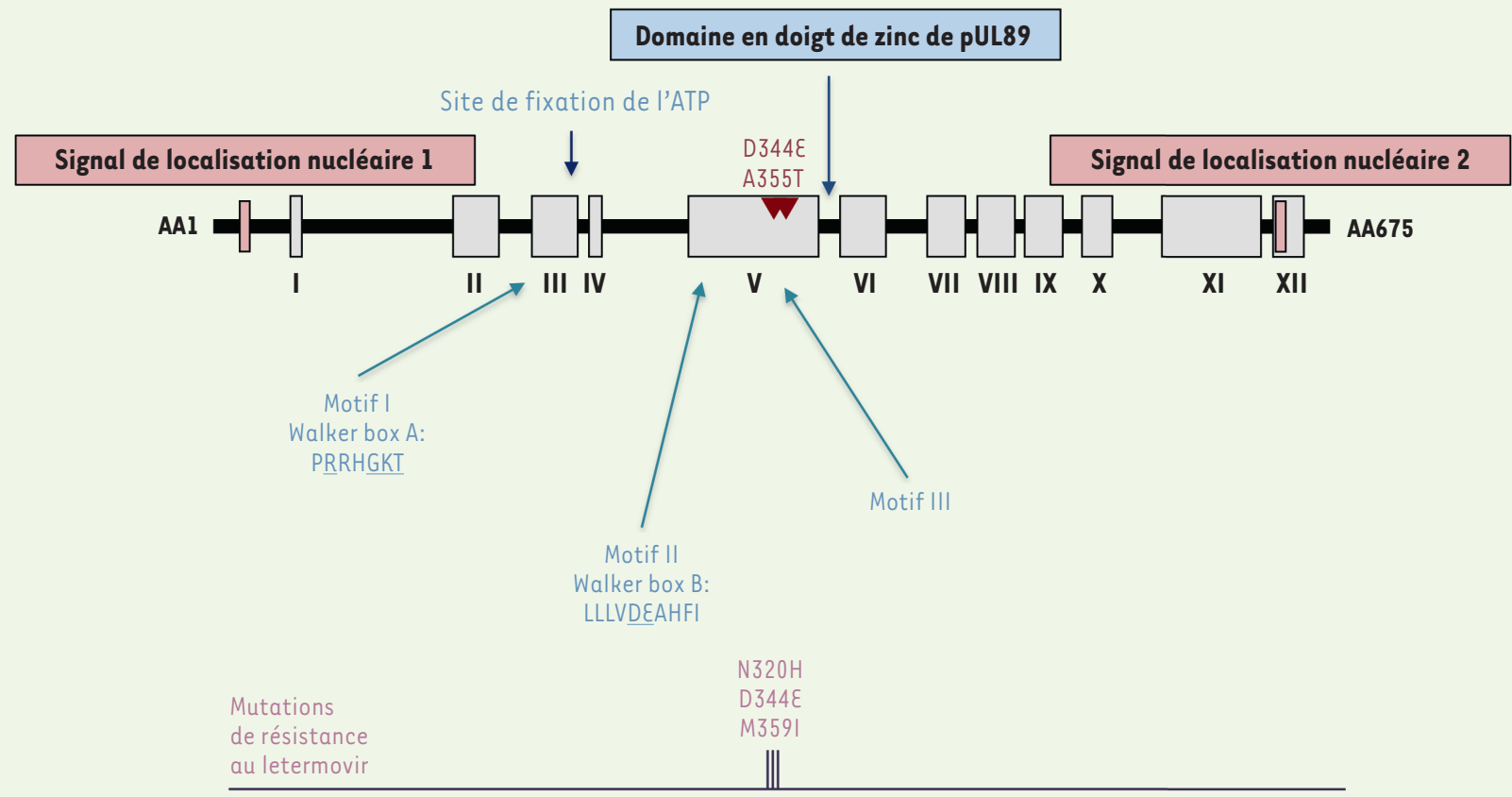

Figure 3. Régions conservées de la sous-unité pUL89. pUL89 est composé de 12 régions conservées (I-XII), avec plusieurs domaines fonctionnels hypothétiques, tels que le site de localisation nucléaire (NLS-1 et NLS-2), un doigt de zinc et un site de liaison à l'ATP. Les acides aminés soulignés sont des résidus impliqués dans les activités des domaines. Les positions des acides aminés associées à la résistance in vitro au letermovir sont indiquées. La position des mutations associées à une résistance au benzimidazole (D344E et A355T) est indiquée en rouge (adaptée de [33]).

portail $^{2}$ pUL104 via sa partie C-terminale. L'activité du complexe dépend de l'hydrolyse de I'ATP qui implique uniquement pUL56 [18] et une mutagenèse dirigée sur le site de liaison de I'ATP de pUL56 $\left({ }_{709} \mathrm{YNETFGK}_{716}\right)$ a montré que la glycine 714 et la lysine 715 étaient essentielles pour cette activité [24].

pUL56 appartiendrait à la famille des «LAGLIDADG homing endonucleases» (LHE). Nous avons en effet mis en évidence, dans la partie $\mathrm{N}$-terminale de la protéine, un motif LATLNDIERFL se rapprochant du motif LAGLIDADG des LHE, et un motif de liaison à I'ADN (doigt de zinc). Par mutagenèse dirigée, nous avons caractérisé les acides aminés potentiellement impliqués dans la dimérisation de pUL56 et dans son activité nucléase, nécessaire au clivage des concatémères d'ADN, et activités essentielles à la réplication virale [25]. Bien que pUL56 soit la protéine présentant le plus grand nombre de fonctions et de zones d'interactions parmi les protéines du complexe, peu de données sont disponibles quant à son interaction avec les autres protéines du complexe et aucune structure cristalline n'a pu être obtenue. Cette absence de données structurales limite ainsi le développement de nouveaux antiviraux.

${ }^{2}$ L'encapsidation nécessite la formation de canaux dans la capside virale. Ces canaux sont réalisés par des protéines virale appelées protéines portail.
La petite sous-unité pUL89

La petite sous-unité du complexe terminase du CMVH, la protéine pUL89, est une protéine de 674 acides aminés codée par un gène de 2025 pb situé dans le segment unique long du génome du CMVH. Différents motifs conservés ont été mis en évidence in silico (Figure 3). On retrouve notamment un motif de fixation de I'ATP $\left({ }_{156} E P F P_{159}\right)$, un motif de type «Walker $A$ » ou motif I ( ${ }_{213}$ PRRHGKT $_{219}$ ), un motif de type «Walker B » ou motif II ( ${ }_{305}$ LLLVDEAHFI $_{314}$ ), et le motif III $\left({ }_{337} \mathrm{SST}_{339}\right.$ ) [26]. Aucune réelle activité enzymatique n'a été mise en évidence pour pUL89. Néanmoins, il a été montré en 2002 que pUL89 augmentait l'activité ATPasique de pUL56 lors de la formation du complexe [18]. La séquence de vingt acides aminés ${ }_{580}$ GRDKALAVEQFISRFNSGYIK $_{600}$ située dans la partie C-terminale de pUL89 serait suffisante pour son interaction avec pUL56 [22]. La structure de ce court domaine a été résolue sous forme d'une hélice alpha exposée [27].

La structure cristalline de la région C-terminale de pUL89 a également permis d'identifier trois résidus conservés (Lysine 583, Alanine 586 et Asparagine 595), participant probablement au domaine d'interaction entre pUL89 et pUL56 [28]. Bien qu'aucune preuve expérimentale n'ait été obtenue, deux domaines NLS 
putatifs ont été proposés pour permettre la translocation nucléaire de pUL89 [26]. Néanmoins, pUL89 ne pourrait rejoindre le noyau qu'associée à pUL56 [29].

\section{La sous-unité pUL51}

La protéine pUL51, composée de 157 acides aminés et codée par un gène de 474 pb situé dans le segment unique long du génome de CMVH, a été récemment identifiée comme étant un partenaire de pUL56 et pUL89, nécessaire au clivage de I'ADN viral [7,21]. pUL51, par son extrémité $\mathrm{C}$-terminale, interagit avec les sous-unités pUL56 et pUL89 et assure leur localisation nucléaire [30]. L'association des sous-unités pUL56 et pUL89 à pUL51, constituant le complexe terminase entièrement assemblé, protège de sa dégradation par le protéasome de la cellule hôte [21]. Peu de données sont, à ce jour, disponibles sur pUL51 et aucune structure cristalline n'a été obtenue.

Les partenaires du complexe terminase récemment identifiés L'étape d'encapsidation du génome viral semble impliquer davantage de protéines que les sous-unités pUL56, pUL89 et pUL51. Au moins trois protéines supplémentaires, pUL52, pUL77 et pUL93, participeraient également au processus. pUL52 est ainsi nécessaire pour cliver les concatémères d'ADN, mais elle n'intervient pas pour la localisation nucléaire des sous-unités du complexe terminase [31]. pUL93 interagit avec pUL77 et les composants du complexe de sortie nucléaire, pUL50, pUL53 et pUL97. Ces protéines joueraient un rôle essentiel dans l'étape d'encapsidation du génome du CMVH, mais leurs fonctions biologiques restent mal caractérisées et leur structure tridimensionnelle n'a pas encore été résolue.

\section{Le complexe terminase : nouvelle cible thérapeutique et développement du letermovir}

L'intérêt grandissant pour le complexe terminase s'explique par ses caractéristiques qui en font une cible virale de choix. En effet, ce complexe hétéro-oligomérique indispensable à la réplication virale ne présente pas d'homologues dans les cellules. De plus, ses différentes fonctions enzymatiques représentent une multitude de cibles potentielles pour de futures molécules antivirales [33]. Outre l'induction de changements de conformation affectant l'orientation du complexe, l'inhibition directe de l'interaction entre les différentes sous-unités ou la protéine portail pUL104 peut être envisagée. D'autres voies peuvent également être explorées, comme l'inhibition de l'activité nucléase ou la liaison de I'ATP à pUL56.

Le letermovir, une molécule de la classe des quinazolines, cible l'encapsidation du génome viral en interagissant avec les protéines du complexe terminase [34]. Les données précliniques obtenues pour cette molécule révèlent sa très forte activité antivirale et, comparée aux autres molécules antivirales, une faible toxicité [35]. Les essais cliniques de phase I et II ont confirmé ces données précliniques [36]: la molécule est bien tolérée et sans effet secondaire notable. Comparé à un placebo, le letermovir présente une forte activité prophylaxique chez les receveurs de greffes de cellules souches hématopoïétiques.
La réduction de l'incidence de l'infection dépend de la dose utilisée, la dose maximale $(480 \mathrm{mg})$ montrant la meilleure efficacité [37].

Malgré l'efficacité de cette molécule, la possibilité de l'émergence de résistances a été démontrée in vitro. Les premières mutations du virus observées en réponse au letermovir ont été localisées dans la partie $\mathrm{N}$-terminale de pUL56, à proximité du potentiel motif doigt de zinc $[38,39]$. Des mutations de pUL89 (D344E, N320H et M359I) ont également été identifiées, conférant une faible résistance du virus au letermovir [40]. De même, une potentielle mutation (P91S) de résistance a été détectée in vitro au sein de pUL51 [41]. La caractérisation phénotypique du virus muté a confirmé que cette mutation conférait, à elle seule, une résistance au letermovir 2,4 fois supérieure par rapport à la souche de référence et, lorsque combinée avec des mutations de pUL56 (S229F ou R369M), multipliait par 3 à 6 le niveau de résistance. Lors de l'essai clinique de phase III, certaines mutations affectant les trois sous-unités principales du complexe terminase ( $p U L 56$, pUL89, pUL51) ont été détectées chez des patients ne répondant pas au letermovir $[42,43]$, suggérant que le letermovir inhibe une fonction biologique nécessitant l'interaction des trois sous-unités, telle que la fixation aux séquences «pac», le clivage ou la translocation d'une unité de génome dans une pro-capside. $\varepsilon n$ raison des connaissances limitées sur la structure des protéines du complexe terminase, déterminer si les mutations associées à la résistance dans les trois gènes codant les sous-unités correspondent à des sites d'interaction avec le letermovir reste difficile.

\section{Vers une meilleure compréhension du mécanisme d'action du letermovir}

Contrairement à d'autres inhibiteurs de l'encapsidation du génome viral, le letermovir est spécifique du CMVH. L'absence d'effet vis-à-vis d'autres herpèsvirus, qui se réactivent également chez les patients immunodéprimés, limite donc le spectre d'activité du letermovir en clinique [44]. Afin de mieux comprendre cette spécificité, nous avons étudié la localisation des mutations associées à la résistance au letermovir, liées aux domaines conservés de pUL56 identifiés par homologie [45]. L'impact potentiel des polymorphismes de pUL56 sur la sensibilité du virus au letermovir a récemment été étudié en utilisant un panel de 74 isolats cliniques [46]. La majorité des polymorphismes qui ont ainsi été identifiés sont situés dans les deux régions variables de pUL56 mais aucun dans la région de résistance au letermovir (AA230 à AA370). Cela 


\section{ALPHAHERPES}

HHV_1

HHV 2

CeHV 1

CeHV 2

$\mathrm{EHV} \overline{1}$

GaHV 3

$\mathrm{MeHV} 1$

HHV $\overline{3}$

SUID

GaHV_1

GaHV_2

\section{BETAHERPES}

AD169

HHV 6 B

HHV ${ }^{-6}{ }^{-}{ }^{\mathrm{A}} \mathrm{GS}$

CCMV

RhCMV

MCMV

RCMV

\section{GAMMAHERPES}

HHV 8

HHV_4_1

$\mathrm{HHV} 4-2$
LDANELRRYLPHAAGLSDADRARALSVLDHAL

LDANELRRYLPHAAGLSDAARARALCVLDQAL LDDNELRKNLPYAGGFSEAARARALRVLDQAL VDEDDLLKNLPYAGGHAPQRLNALCEDLRHAR VANI DIARYLPHASGVASERRAAAEAALRALE VHPDDMVAHI PHAACLSDEKRSVVQAALSLIR VDPDDMVAHLPHATYILPDKLAVATTALNHIN VDVDDI IRCLPYI PDVPDIKRQSAVEALRTLQ VDPED AKNLPHVHGLDEARRGRALAALAAVD VSTDDLKRHLPFLRDVDPEKLKNVLSNETRIE VNSDDLLTHLPHA YVPDDKRTTAQTALDVIQ

VNIQTVEQDLLDLTTRI PHLA-GVLSALKSLF
KNIETIEKDLGEAILNYGTIQ-SVVTEIKKIF
KNIETIEKDLGEAILNYGTVQ-SVATEIKKIF
MDAQAVEQDMGDIAIRAPSVK-GIIRAIKSLA
MDAQAVEQDMGDIAIRAPSVK-GIIRAIKSLF
MDAQAVEQDMGDIAIRAPSVK-GIIRAIKSLA
TDAQTVEQDLGEVAIRAPRVK-SIIRSLKNLA

G---LFENELKQLGLQTPESI-----PTTPCQ

G---LFENEVRQAGLGHLLEAEEKARPGGPEE

G---LFENEVRQAGLGHLLEAEEKARPGGPEE
ASNLTALAR

ADNLNALAQ

ADNLTELAR

GRRLADLEE

TENLETLDK

ADNLEDLAF

ADNLEQVVA

VDNLDVLNE

THNLDDLER

DKDLSELFK

ADNLEALVS

YHQLCRALM
CHTLYKQLQ
CHTLYKQLQ
SHSLWKTLR
YHHLCRVLM
SHSLWKTLR
SHGLWKALR

QATSPDDQIEALI QATSPDDQIEALI QATSPEDQIEALI PRLPPETRMVALI CAVSPDDHIEALI RAVAPDDIIEALI RTAAPDDIIEALI RSSSPDSQIEALI CAASPDERLEALI LATSPDTEIAALI RAAAPDDIIEALI

VFAAPNRIIDLIT IYVSPSRLIDLVT TYSSPKNS IYLKS IFAAPGKVVDMIT VFAAPSRIIDLIT IFAAPGKVVDMIT ISAAPGKVVDMIT

CSHLASLFT HSRLSALFA HSRLSALFA
LFSSVEDTIESLQ

VYSSSGDAVEALK

VYSSSGDAVEALK

Région VI - ع356-|374

Figure 4. Alignement des séquences de la région conservée pUL56 VI de 21 herpèsvirus. La numérotation des séquences est conforme à celle des résidus de la souche de référence AD169. Les résidus de CMVH impliqués dans la résistance au letermovir sont représentés par des lettres blanches sur un fond noir. Les résidus des virus contre lesquels le letermovir a été testé sont représentés par des lettres noires sur fond gris.

suggère fortement que tous les résidus connus pour être associés à une résistance au letermovir sont fortement conservés, sans toutefois être toujours situés dans des domaines conservés de la protéine.

La substitution de l'arginine 369 (R369M/G/S) qui confère une résistance de haut niveau au letermovir, est située dans la région conservée VI de la protéine virale [47]. Ce résidu n'a cependant été retrouvé que dans la protéine du CMVH et de l'HHV-6_B (Figure 4). Chez d'autres herpèsvirus, cette arginine est substituée par un aspartate (HSV-1, HSV-2, HHV-4_l et HHV-4_2), une asparagine (HHV-6A-GS), une sérine (HHV-3), ou une lysine (MCMV et RCMV). Ces substitutions différentes pourraient expliquer le manque d'efficacité du letermovir pour ces herpèsvirus. Contrairement à d'autres herpèsvirus, HHV-6_B possède également une arginine en position 369, mais la sensibilité de ce virus au letermovir n'a pas été testée. Le manque d'activité du letermovir associé à la substitution de la sérine peut résulter de la différence de taille de cet acide aminé par rapport à celle de l'arginine. Pour l'aspartate, ce défaut reposerait sur la présence d'une charge négative. La présence d'une chaîne latérale plus courte peut également expliquer le manque d'activité que l'on observe dans le cas d'une substitution par l'arginine ou par l'asparagine. Pour mieux comprendre l'impact de l'acide aminé R369 sur l'interaction potentielle de la pro- téine virale avec le letermovir, nous avons construit un modèle théorique du petit fragment (résidus 224 à 450 , région VI comprise) à l'aide du serveur CPHmodels-3.2 (Figure 5). L'acide aminé R369 (en rouge dans la figure) est situé dans une boucle. Les analyses de la région riche en leucine proche de cette boucle ont permis de prédire l'existence de deux hélices alpha qui pourraient constituer un «leucine zipper». Les leucines sont conservées au sein des bêta-herpèsvirus ou substituées par une isoleucine ou une valine. Le mécanisme d'action du letermovir pourrait reposer sur son interaction avec plusieurs résidus situés dans la boucle (Figure 5).

La plupart des résidus impliqués dans la résistance du letermovir ont été retrouvés chez d'autres herpèsvirus, tels que HSV-1 ou HSV-2. Par exemple, la substitution touchant la leucine 24l (L24lP dans le CMVH) a également été identifiée chez tous les alphaherpèsvirus. Cette leucine est substituée par une glycine chez d'autres bêta-herpèsvirus.

Il pourrait être intéressant d'étudier la capacité relative d'émergence de ces mutations dans un contexte 
A

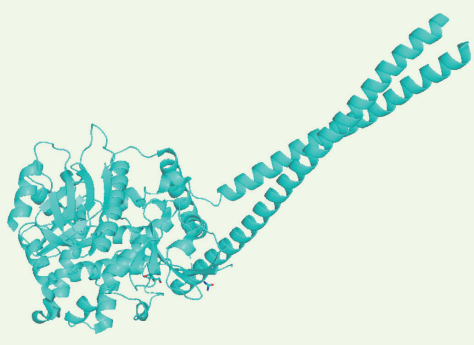

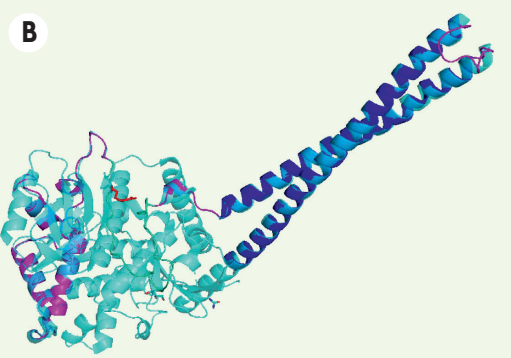

C
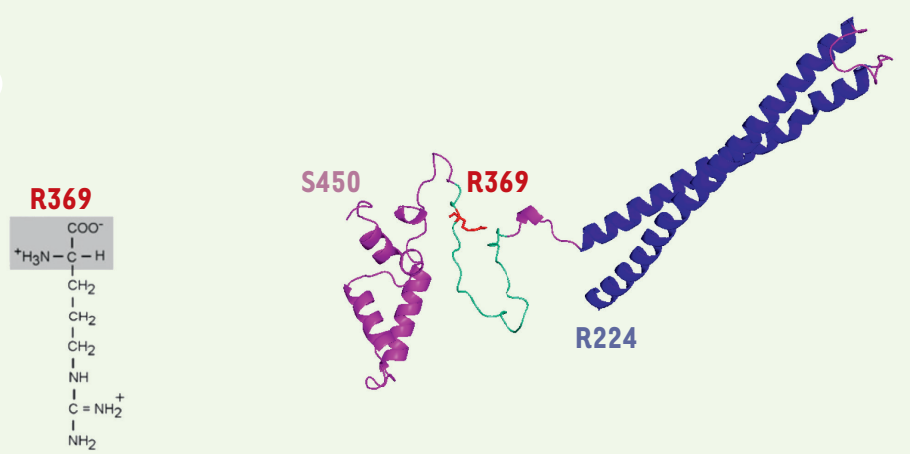

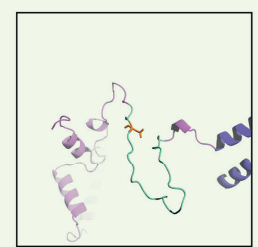

D369

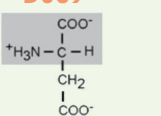

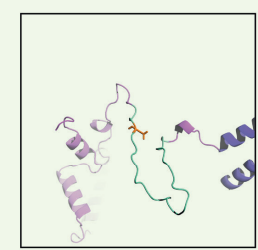

N369

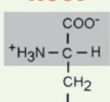

${ }_{\mathrm{H}_{2} \mathrm{~N}^{\mathrm{N}}}^{\mathrm{C}} \stackrel{\mathrm{C}}{\mathrm{C}}$
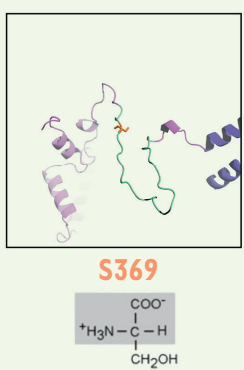

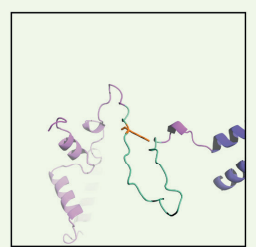

K369

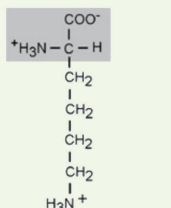

Figure 5. Modélisation par homologie d'un segment de pUL56 (résidus 224 à 450) mettant en évidence le rôle du résidu R369 dans le mécanisme d'action du letermovir. A. Structure de la moésine de Spodoptera frugiperda (PDB : 211K.A). B. Superposition de la structure de la moésine de Spodoptera frugiperda et de la structure théorique de pUL56(224-450). C. Structure théorique de pUL56(224-450) obtenue grâce au serveur CPHmodels-3.2. Le résidu R369 sur la boucle (en vert) est représenté en rouge sur le modèle théorique. Deux hélices alpha représentant une structure « Leucine zipper » sont représentées en bleu.

clinique. Ces mutations existant chez d'autres herpèsvirus, elles pourraient représenter un avantage et seraient sélectionnées lors de la réplication virale en contexte clinique. La divergence des séquences protéiques, et donc la conformation des sous-unités du complexe terminase, pourrait probablement expliquer la spécificité du letermovir pour le CMVH.

\section{Nouvelles cibles thérapeutiques et nouveaux antiviraux}

À côté des inhibiteurs de l'encapsidation, de nouveaux antiviraux sont en cours de développement afin de pallier la toxicité des traitements disponibles et l'émergence de mutations provoquant une résistance. De nombreuses voies sont explorées: des inhibiteurs de la polymérase pUL54 ou des molécules ciblant des voies de la réplication cellulaire interférant avec la réplication virale (artésunate et dérivés, maribavir). Parmi les inhibiteurs de la polymérase, le brincidofovir est une prodrogue du cidofovir conjugué à un lipide afin d'améliorer sa biodisponibilité. Il a été proposé en prévention de la maladie à CMVH dans un essai clinique de phase III. Le bénéfice de son utilisation en thérapeutique, lors d'infections par le $\mathrm{CMVH}$, a été récemment montré, bien que les mutations associées à une résistance à ce traitement, touchant le gène UL54, puissent limiter son 
efficacité thérapeutique [48]. Le brincidofovir ne permet cependant pas de réduire significativement les infections/maladies à CMV chez les greffés de cellules souches hématopoïétiques. II présente également des effets indésirables digestifs, ne permettant pas d'envisager son utilisation en clinique [49]. L'artésunate, un dérivé semi-synthétique de l'artémisine, est habituellement préconisé dans le traitement des accès palustres graves [50-52]. Il a été testé en prévention de la maladie à CMVH, mais son utilisation n'a pas été poursuivie en raison d'une efficacité médiocre sur la charge virale [53]. L'artésunate semble interférer avec la voie de signalisation impliquant NF- $\kappa B$ (« nuclear factor-kappa $B »)$ en inhibant la fixation de sa sous-unité RelA/p65 à l'ADN cellulaire [54]. Des dérivés de l'artesunate sont actuellement évalués dans des essais pré-cliniques [55].

Le maribavir ${ }^{3}$ est un dérivé benzimidazolé lévogyre du BDCRB ${ }^{4}$, qui cible la kinase virale pUL97. II présente une faible toxicité et une bonne biodisponibilité [56]. Lors des premiers essais cliniques de phase III, son efficacité en prophylaxie à faibles doses n'a pas été démontrée [57]. Cependant, son efficacité à fortes doses en cas d'infection résistante aux anti-polymérases [58] a justifié la poursuite de son développement clinique [59].

De nouvelles cibles thérapeutiques ont été mises en évidence pour cibler les alpha-herpèsvirus : le complexe hélicase-primase ciblé par le pritelevir (HSV-1, HSV-2 et VZV) et l'amenamevir (HSV-1 et HSV-2). Ces molécules ne présentent cependant aucune activité contre le $\mathrm{CMVH}$, les domaines conservés de l'hélicase-primase du CMVH présentant des différences significatives avec ceux des alpha-herpèsvirus sensibles [60]. Ce complexe pourrait ainsi devenir une cible antivirale de choix pour le traitement de l'infection par le CMVH.

\section{Conclusion}

Étudier la relation entre structure et fonction des protéines virales pourrait permettre de mieux appréhender le fonctionnement de complexes viraux impliqués dans le cycle de réplication du virus. Ces connaissances pourraient constituer un point de départ essentiel à l'identification de nouvelles cibles virales et, par conséquent, au développement de molécules à visée préventive, thérapeutique ou diagnostique. Elles permettraient également de mieux comprendre le mécanisme d'action de molécules en développement. $\diamond$

\section{SUMMARY}

The terminase complex, a relevant target for the treatment of HCMV infection

Human cytomegalovirus (HCMV) is an important ubiquitous opportunistic pathogen that belongs to the betaherpesviridae. Primary HCMV infection is generally asymptomatic in immunocompetent individuals. In contrast, HCMV infection causes serious disease in immunocompromised patients and is the leading cause of congenital viral infection. Although they are effective, the use of conventional molecules is

${ }^{3}$ 2-isopropylamino-5,6-dichloro-1-( $\beta$-L-ribofuranosyl)benzimidazole.

${ }^{4}$ l-H- $\beta$-D-ribofuranoside-2-bromo-5,6-dichlorobenzimidazole. limited by the emergence of resistance and by their toxicity. New antivirals targeting other replication steps and inducing fewer adverse effects are therefore needed. During HCMV replication, DNA packaging is performed by the terminase complex, which cleaves DNA to package the virus genome into the capsid. With no counterpart in mammalian cells, these terminase proteins are ideal targets for highly specific antivirals. A new terminase inhibitor, letermovir, recently proved effective against HCMV in phase III clinical trials. However, its mechanism of action is unclear and it has no significant activity against other herpesvirus or non-human CMV. $\diamond$

\section{LIENS D'INTÉRÊT}

Les auteurs déclarent n'avoir aucun lien d'intérêt concernant les données publiées dans cet article.

\section{RÉFÉRENCES}

1. Rubin RH. Prevention of cytomegalovirus infection in organ transplant recipients. Transpl Infect Dis $2000 ; 2: 99-100$.

2. Pereyra F, Rubin RH. Prevention and treatment of cytomegalovirus infection in solid organ transplant recipients. Curr Opin Infect Dis $2004 ; 17$ : 357-61.

3. Andouard D, Mazeron MC, Ligat G, et al. Contrasting effect of new HCMV pUL54 mutations on antiviral drug susceptibility: Benefits and limits of 3D analysis. Antiviral Res 2016 ; 129 : 115-9.

4. Leruez-Ville M, Ghout I, Bussières L, et al. In utero treatment of congenital cytomegalovirus infection with valacyclovir in a multicenter, open-label, phase II study. Am J Obstet Gynecol 2016 ; 215 : 462.el-el0.

5. McVoy MA, Adler SP. Human cytomegalovirus DNA replicates after early circularization by concatemer formation, and inversion occurs within the concatemer. J Virol $1994 ; 68: 1040-51$.

6. Scheffczik H, Savva CGW, Holzenburg A, et al. The terminase subunits pUL56 and pUL89 of human cytomegalovirus are DNA-metabolizing proteins with toroidal structure. Nucleic Acids Res 2002 ; 30 : 1695-703.

7. Borst EM, Kleine-Albers J, Gabaev I, et al. The human cytomegalovirus UL51 protein is essential for viral genome cleavage-packaging and interacts with the terminase subunits pUL56 and pUL89. J Virol 2013; $87: 1720-32$.

8. Borst $\varepsilon M$, Wagner $K$, Binz $A$, et al. The essential human cytomegalovirus gene UL52 is required for cleavage-packaging of the viral genome. J Virol 2008 ; $82: 2065-78$.

9. Borst $\varepsilon M$, Bauerfeind R, Binz A, et al. The Essential human cytomegalovirus proteins pUL77 and pUL93 are structural components necessary for viral genome encapsidation. J Virol 2016; $90: 5860-75$.

10. DeRussy BM, Tandon R. Human cytomegalovirus pUL93 is required for viral genome cleavage and packaging. J Virol $2015 ; 89: 12221-5$.

11. Köppen-Rung P, Dittmer A, Bogner $\varepsilon$. Intracellular distributions of capsidassociated pUL77 of HCMV and interactions with packaging proteins and pUL93. J Virol $2016 ; 90: 5876-85$.

12. DeRussy BM, Tandon R. Human cytomegalovirus pUL 93 is required for viral genome cleavage and packaging. J Virol $2015 ; 89$ : 12221-5.

13. Bogner $\varepsilon$. Human cytomegalovirus terminase as a target for antiviral chemotherapy. Rev Med Virol 2002 ; 12 : 115-27.

14. Champier G, Couvreux A, Hantz $S$, et al. Putative functional domains of human cytomegalovirus pUL56 involved in dimerization and benzimidazole D-ribonucleoside activity. Antivir Ther 2008 ; 13 : 643-54.

15. Addison C, Rixon FJ, Preston VG. Herpes simplex virus type 1 UL28 gene product is important for the formation of mature capsids. J GenVirol 1990 ; $71: 2377-84$

16. Bogner $\varepsilon$, Reschke $M$, Reis $B$, et al. Identification of the gene product encoded by ORF UL56 of the human cytomegalovirus genome. Virology 1993 ; $196: 290-3$.

17. Savva CGW, Holzenburg A, Bogner $\varepsilon$. Insights into the structure of human cytomegalovirus large terminase subunit pUL56. FEBS Lett $2004 ; 563$ : 135-40.

18. Hwang J-S, Bogner $\varepsilon$. ATPase activity of the terminase subunit pUL56 of human cytomegalovirus. J Biol Chem 2002 ; 277 : 6943-8. 


\section{RéFÉRENCES}

19. Bogner $\varepsilon$, Radsak K, Stinski MF. The gene product of human cytomegalovirus open reading frame UL56 binds the pac motif and has specific nuclease activity. J Virol $1998 ; 72: 2259-64$.

20. Giesen K, Radsak K, Bogner $\varepsilon$. Targeting of the gene product encoded by ORF UL56 of human cytomegalovirus into viral replication centers. FEBS Lett $2000 ; 471: 215-8$.

21. Neuber $S$, Wagner $K$, Goldne T, et al. Mutual interplay between the human cytomegalovirus terminase subunits pUL51, pUL56, and pUL89 promotes terminase complex formation. J Virol 2017 ; 91. pii: e02384-16.

22. Thoma $C$, Borst $\varepsilon$, Messerle $M$, et al. Identification of the interaction domain of the small terminase subunit pUL89 with the large subunit pUL56 of human cytomegalovirus. Biochemistry $2006 ; 45: 8855-63$.

23. Ligat $\mathrm{G}$, Jacquet $\mathrm{C}$, Chou $\mathrm{S}$, et al. Identification of a short sequence in the HCMV terminase pUL56 essential for interaction with pUL89 subunit. Sci Rep $2017 ; 7: 8796$

24. Scholz B, Rechter S, Drach JC, et al. Identification of the ATP-binding site in the terminase subunit pUL56 of human cytomegalovirus. Nucleic Acids Res $2003 ; 31$ : 1426-33.

25. Ligat $G$, Couvreux A, Cazal R, et al. Highlighting of a LAGLIDADG and a zing finger motifs located in the pUL56 sequence crucial for HCMV replication. Viruses $2019 ; 11$.

26. Champier G, Hantz S, Couvreux A, et al. New functional domains of human cytomegalovirus pUL 89 predicted by sequence analysis and three-dimensional modelling of the catalytic site DEXDc. Antivir Ther $2007 ; 12: 217-32$

27. Couvreux A, Hantz S, Marquant $R$, et al. Insight into the structure of the pUL89 C-terminal domain of the human cytomegalovirus terminase complex. Proteins $2010 ; 78: 1520-30$.

28. Nadal M, Mas PJ, Blanco AG, et al. Structure and inhibition of herpesvirus DNA packaging terminase nuclease domain. Proc Natl Acad Sci USA 2010 ; 107 : 16078-83.

29. Wang JB, Zhu Y, McVoy MA, et al. Changes in subcellular localization reveal interactions between human cytomegalovirus terminase subunits. Virol J $2012 ; 9: 315$.

30. Neuber S, Wagner K, Messerle M, et al. The C-terminal part of the human cytomegalovirus terminase subunit PUL51 is central for terminase complex assembly. J Gen Virol 2017 ; 99 : 119-34.

31. Borst EM, Wagner K, Binz A, et al. The essential human cytomegalovirus gene UL52 is required for cleavage-packaging of the viral genome. J Virol $2008 ; 82: 2065-78$.

32. DeRussy BM, Boland MT, Tandon R. Human cytomegalovirus pUL 93 links nucleocapsid maturation and nuclear egress. J Virol $2016 ; 90: 7109-17$.

33. Ligat G, Cazal R, Hantz S, et al. The human cytomegalovirus terminase complex as an antiviral target: a close-up view. FEMS Microbiol Rev 2018 ; 42 : 137-45.

34. Goldner T, Hewlett G, Ettischer N, et al. The novel anticytomegalovirus compound AIC246 (Letermovir) inhibits human cytomegalovirus replication through a specific antiviral mechanism that involves the viral terminase. J Virol $2011 ; 85: 10884-93$

35. Lischka P, Hewlett G, Wunberg T, et al. In vitro and in vivo activities of the novel anticytomegalovirus compound AIC246. Antimicrob. Agents Chemother $2010 ; 54: 1290-7$.

36. Stoelben S, Arns W, Renders L, et al. Preemptive treatment of cytomegalovirus infection in kidney transplant recipients with letermovir: results of a phase 2 a study. Transplant $2014 ; 27: 77-86$

37. Chemaly RF, Ullmann AJ, Stoelben $S$, et al. Letermovir for cytomegalovirus prophylaxis in hematopoietic-cell transplantation. N Engl J Med 2014 ; 370 : 1781-9.

38. Piret J, Goyette N, Boivin G. Drug susceptibility and replicative capacity of multi-drug resistant recombinant human cytomegalovirus harboring mutations in UL56 and UL54 genes. Antimicrob Agents Chemother 2017 ; 61. pii: e01044-17.

39. Goldner T, Hempel C, Ruebsamen-Schaeff H, et al. Geno- and phenotypic characterization of human cytomegalovirus mutants selected in vitro after letermovir (AIC246) exposure. Antimicrob. Agents Chemother $2014 ; 58: 610-3$

40. Chou S. Comparison of cytomegalovirus terminase gene mutations selected after exposure to three distinct inhibitor compounds. Antimicrob Agents Chemother $2017 ; 61$.

41. Chou S. A third component of the human cytomegalovirus terminase complex is involved in letermovir resistance. Antiviral Res $2017 ; 148: 1-4$

42. Komatsu TE, Hodowanec AC, Colberg-Poley AM, et al. In-depth genomic analyses identified novel letermovir resistance-associated substitutions in the cytomegalovirus UL56 and UL89 gene products. Antiviral Res 2019; 169 : 104549

43. Douglas CM, Barnard R, Holder D, et al. Letermovir resistance analysis in a clinical trial of cytomegalovirus prophylaxis for hematopoietic stem cell transplant recipients. J Infect Dis 2019 ; pii: jiz577.

44. Marschall M, Stamminger T, Urban A, et al. In vitro evaluation of the activities of the novel anticytomegalovirus compound AIC246 (letermovir) against herpesviruses and other human pathogenic viruses. Antimicrob. Agents Chemother $2012 ; 56: 1135-7$

45. Champier G, Couvreux A, Hantz S, et al. Putative functional domains of human cytomegalovirus pUL56 involved in dimerization and benzimidazole D-ribonucleoside activity. Antivir Ther $2008 ; 13: 643-54$.

46. Lischka P, Michel D, Zimmermann H. Characterization of cytomegalovirus breakthrough events in a letermovir (AIC246, MK 8228) phase 2 prophylaxis trial. J Infect Dis $2015 ; 213: 23-30$.

47. Goldner T, Hempel C, Ruebsamen-Schaeff H, et al. Geno- and phenotypic characterization of human cytomegalovirus mutants selected in vitro after letermovir (AIC246) exposure. Antimicrob Agents Chemother $2014 ; 58$ : 6103

48. Vial R, Zandotti C, Alain S, et al. Brincidofovir use after foscarnet crystal nephropathy in a kidney transplant recipient with multiresistant cytomegalovirus infection. Case Rep Transplant 2017 ; 2017 : 3624146.

49. Marty FM, Winston DJ, Chemaly RF, et al. A randomized, double-blind, placebo-controlled phase 3 trial of oral brincidofovir for cytomegalovirus prophylaxis in allogeneic hematopoietic cell transplantation. Biol Blood Marrow Transplant $2019 ; 25: 369-81$.

50. Kaptein SJF, Efferth T, Leis M, et al. The anti-malaria drug artesunate inhibits replication of cytomegalovirus in vitro and in vivo. Antiviral Res $2006 ; 69: 60-9$.

51. Chou S, Marousek G, Auerochs S, et al. The unique antiviral activity of artesunate is broadly effective against human cytomegaloviruses including therapy-resistant mutants. Antiviral Res $2011 ; 92: 364-8$.

52. Germi R, Mariette $C$, Alain $S$, et al. Success and failure of artesunate treatment in five transplant recipients with disease caused by drug resistant cytomegalovirus. Antiviral Res $2014 ; 101: 57-61$.

53. Wolf DG, Shimoni A, Resnick IB, et al. Human cytomegalovirus kinetics following institution of artesunate after hematopoietic stem cell transplantation. Antiviral Res $2011 ; 90$ : 183-6.

54. Lyss G, Knorre A, Schmidt TJ, et al. The anti-inflammatory sesquiterpene lactone helenalin inhibits the transcription factor NF-kappaB by directly targeting p65. J Biol Chem $1998 ; 273$ : 33508-16.

55. Hutterer C, Niemann I, Milbradt J, et al. The broad-spectrum antiinfective drug artesunate interferes with the canonical nuclear factor kappa B (NF$\kappa B)$ pathway by targeting RelA/p65. Antiviral Res $2015 ; 124$ : 101-9.

56. Koszalka GW, Johnson NW, Good SS, et al. Preclinical and toxicology studies of 1263 W94, a potent and selective inhibitor of human cytomegalovirus replication. Antimicrob Agents Chemother 2002 ; 46 : 2373-80.

57. Marty FM, Ljungman P, Papanicolaou GA, et al. Maribavir prophylaxis for prevention of cytomegalovirus disease in recipients of allogeneic stem-cell transplants: a phase 3, double-blind, placebo-controlled, randomised trial. Lancet Infect Dis $2011 ; 11$ : 284-92.

58. Alain $S$, Revest $M$, Veyer $D$, et al. Maribavir use in practice for cytomegalovirus infection in French transplantation centers. Transplant Proc 2013 ; 45 : 1603-7.

59. Papanicolaou GA, Silveira FP, Langston AA, et al. Maribavir for refractory or resistant cytomegalovirus infections in hematopoietic-cell or solid-organ transplant recipients: a randomized, dose-ranging, double-blind, phase 2 study. Clin Infect Dis $2019 ; 68: 1255-64$.

60. Ligat $\mathrm{G}, \mathrm{Da} \operatorname{Re} \mathrm{S}$, Alain $\mathrm{S}$, et al. Identification of amino acids essential for viral replication in the HCMV helicase-primase complex. Front Microbiol $2018 ; 9: 2483$

TIRÉS À PART

G. Ligat

Retrouvez toutes les Actualités de la Myologie sur les sites de :

la Société Française de Myologie www.sfmyologie.org la filière de santé neuromusculaire FILNEMUS

www.filnemus.fr 